Perspective

\title{
Towards an Alternate Evaluation of Moisture-Induced Damage of Bituminous Materials
}

\author{
Aboelkasim Diab ${ }^{1}$ (D), Zhanping You ${ }^{2, *}$, Xu Yang ${ }^{3}$ and Mohd Rosli Mohd Hasan ${ }^{4}$ \\ 1 Department of Civil Engineering, Aswan University, Aswan 81542, Egypt; adiab@aswu.edu.eg or \\ daali@mtu.edu \\ 2 Department of Civil and Environmental Engineering, Michigan Technological University, Houghton, \\ MI 49931, USA \\ 3 Department of Civil Engineering, Monash University, Clayton, VIC 3800, Australia; xu.yang@monash.edu \\ 4 School of Civil Engineering, Universiti Sains Malaysia, Engineering Campus, Nibong Tebal Penang 14300, \\ Malaysia; cerosli@usm.my \\ * Correspondence: zyou@mtu.edu; Tel.: +1-906-487-1059
}

Received: 12 September 2017; Accepted: 10 October 2017; Published: 13 October 2017

\begin{abstract}
Moisture-induced damage is widely known to cause multiple distresses that affect the durability of constructed pavements and eventually lead to the costly maintenance of pavement structures. The reliability and practicality of the assessment protocol to evaluate moisture susceptibility of flexible pavements presents a dilemma within the asphalt community that arises from the complexity and interrelation of moisture mechanisms in the asphalt-aggregate system. Researchers worldwide are continuously trying to develop suitable evaluation methods to simulate the combined destructive field-induced effects of moisture in the laboratory to help practitioners identify and alleviate this complex problem. The main objective of this article is to provide insights and highlight the challenges and opportunities of this important topic in order to extend and share knowledge towards finding a realistic assessment protocol of moisture damage in the laboratory. Two scenarios are proposed in this article: (1) a damage rate concept that accounts for the change of mechanical property (e.g., indirect tensile strength) with respect to the conditioning time, and (2) the establishment of a database using a surface free energy concept to help stakeholders select appropriate asphalt-aggregate combinations without the need to run additional moisture susceptibility tests.
\end{abstract}

Keywords: asphalt pavement; moisture damage; damage rate; surface free energy

\section{Outlook}

The distresses related to moisture-induced damage still remain one of the most common but complex issues of bituminous pavements [1,2]. Mixtures that are not properly designed or evaluated against ever increasing traffic loading alongside the expected exposure to moisture in the field could potentially affect the pavement durability that can lead to progressive degradation in the form of several distresses, such as raveling, rutting, or cracking [3-5]. In service life, the bituminous pavement is subjected to different environmental conditions combined with traffic loading, which both affect the durability and life-cycle cost of the constructed structure. Displacement, detachment, spontaneous emulsification, pore-pressure-induced damage, hydraulic scour, $\mathrm{pH}$ instability, and climatic conditions are considered contributing mechanisms associated with moisture damage in the field [6,7]. So far, the moisture damage of asphalt pavements is not well understood, as it is a complex phenomenon that is affected by the physicochemical properties of the constituents forming the asphalt mixture. In the design stage, the asphalt materials are usually characterized for the moisture effect at the macro scale using compacted asphalt samples. Although the evaluation of compacted asphalt mixtures is commonly used in the pavement engineering community, there are several problems still unsolved, and 
different perspectives should be considered towards this issue. During the mixture design, the asphalt mixture must pass the tensile strength ratio (TSR) test to ensure the moisture susceptibility of the designed mixture. As a supporting step, in turn, this perspective article is intended to highlight the current issue of moisture damage evaluation and provide an overview of the opportunities to be considered for future researches.

\section{Challenges}

Various, predominantly empirical, mechanical test methods to evaluate the moisture susceptibility of asphalt mixtures have been developed and are discussed elsewhere [8]. The AASHTO T 283 test method is frequently used by highway agencies worldwide for the sake of evaluation of moisture damage of compacted asphalt concrete mixtures. Basically, in the T 283 test, the TSR, which is calculated as the ratio of the indirect tensile strength (ITS) of conditioned specimens to that for dry specimens, is used to determine the resistance of the asphalt mixture to moisture damage. A minimum TSR of $80 \%$ is usually used as a threshold to ensure that the mixture is moisture-resistant, while the mixture is considered moisture-prone if the TSR is any lower. However, several concerns of this ratio have been discussed $[9,10]$ :

- Does this value guarantee a satisfactory resistance to moisture damage in the long-term? The current practice of the TSR concept is based on short-term aged samples, but the moisture susceptibility is a concern throughout the service life of asphalt pavement.

- What is the proper conditioning method to mimic the moisture damage in real life? Freeze-thaw is used in AASHTO T 283, and the moisture induced stress tester (MIST) was introduced in recent years. Can these methods, or some other method, better mimic real moisture damage?

- As the unconditioned or dry compacted specimens are different from the conditioned or wet specimens, does specimen-to-specimen variability affect the results? Even for the same mixture, the prepared compacted samples would have different distributions of aggregate particles and/or air voids, which affects the measured property. Therefore, the test still lacks repeatability, and its accuracy is questionable.

- Does the saturation level while conditioning affect the repeatability of the results?

- Based on previous studies on the AASHTO T 283 test, a number of successful cases that pass in the laboratory would fail in the field.

\section{Opportunities}

As for suggestions, two scenarios for the evaluation of moisture damage are discussed in this article, the future studies may evaluate.

\subsection{First Scenario}

The moisture-induced damage of asphalt mixtures can be considered as a time-dependent damage due to the combined effects of moisture and traffic loading. The mechanical property $M P(t)$ at time $t$ is affected by the initial mechanical property $M P(0)$ (i.e., dry specimen) and the induced damage $D(t)$ during conditioning. The progressive damage due to moisture and/or traffic loading can be represented as

$$
M P(t)=M P(0)-D(t) .
$$

Based on Equation (1), Figure 1 depicts a schematic of the mechanical property (such as ITS) with respect to the time of conditioning. As examples, the figure compares possible trends of two different mixes. 


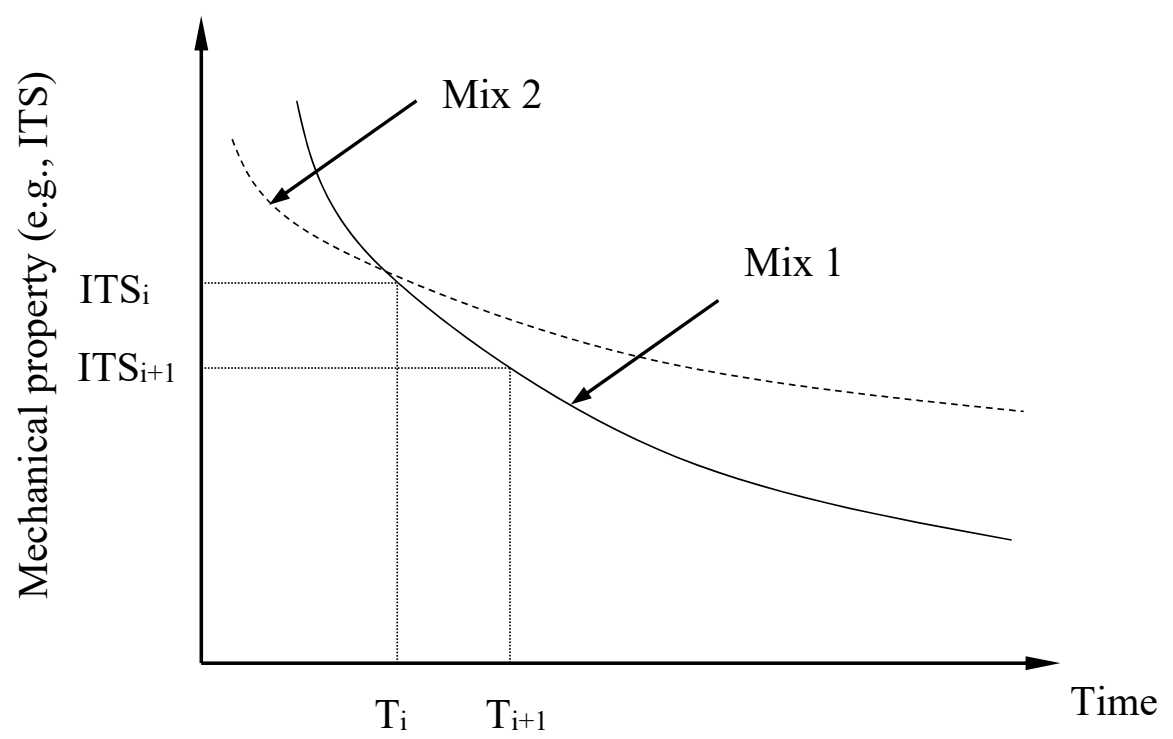

Figure 1. Mechanical property as a function of conditioning time due to the effect of environmental conditions and traffic loading.

This method should involve the conditioning and evaluation steps. In the conditioning step, realistic or field-like conditions should be adopted to induce reasonable effects of moisture and traffic. In a recently published work [9], the authors suggested a conditioning procedure by exposing compacted asphalt specimens to a temperature as high as the highest performance grading (PG) of the used asphalt binder, while the traffic loading was selected based on realistic effects from the literature to simulate the pressure of vehicle wheel on the wet surface. These effects were integrated into the proposed conditioning system in the laboratory. In regard to the evaluation, rather than using a single criterion (i.e., TSR), a model was developed to identify the time-dependent damage of the mixture using the damage rate $\mathrm{D}_{\mathrm{r}}$ concept:

$$
\mathrm{D}_{\mathrm{r}}=\frac{\mathrm{ITS}_{\mathrm{i}}-\mathrm{ITS}_{\mathrm{i}+1}}{\mathrm{~T}_{\mathrm{i}+1}-\mathrm{T}_{\mathrm{i}}}
$$

In this model, one can quantify the moisture damage over time and determine how much the mixture degrades with respect to time of conditioning. Using the concept of damage rate, the moisture effects can be evaluated at different times, which is more promising as the mixture might have different behaviors over time. Although its usefulness to discriminate between insusceptible and moisture susceptible mixtures in a rational manner, the concept necessitates the development of a threshold to help quantitatively perform the judgment.

\subsection{Second Scenario}

The second suggested scenario is beneficial to pavement contractors and can save more time. Under the effects of moisture and traffic, the susceptibility to moisture damage of asphalt-aggregate systems is related to cohesive failure within the matrix and/or adhesive failure at the interface between the asphalt binder and aggregate (Figure 2). 


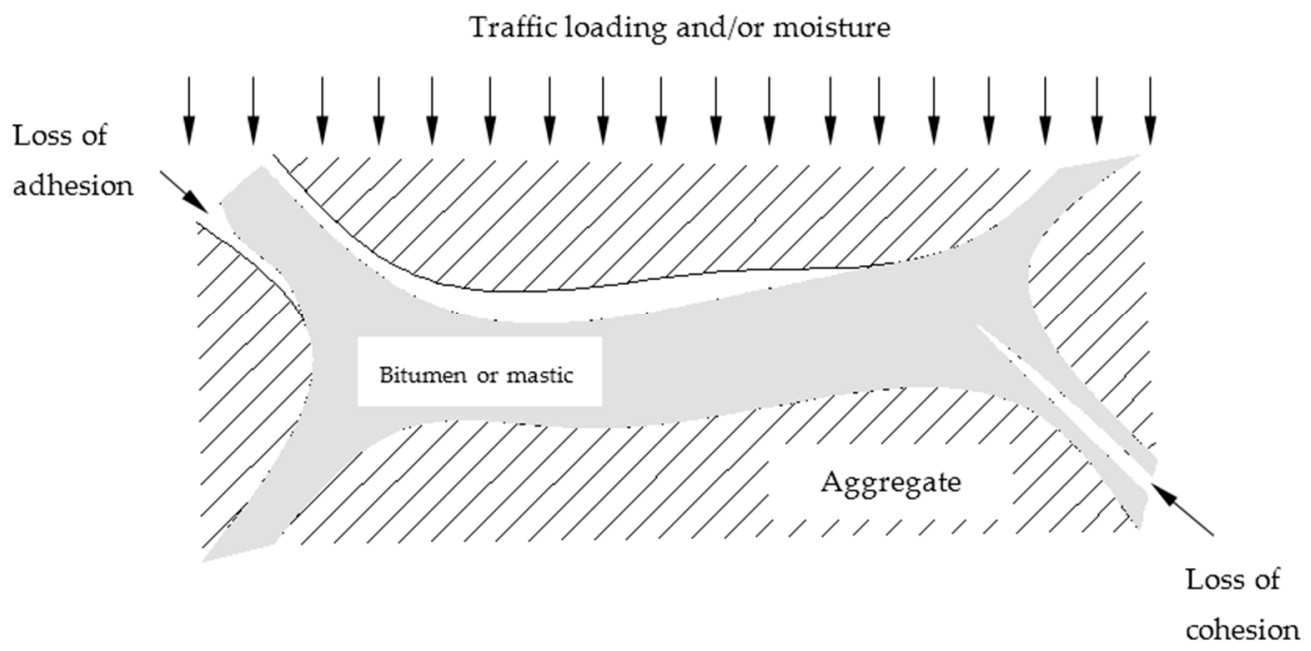

Figure 2. Possible failures due to moisture damage and traffic loading.

The moisture damage of asphalt mixtures can be studied through fundamental approaches to understand the mechanisms causing the susceptibility to moisture damage. Based on the theory of thermodynamics, the surface free energy (SFE) can be used to evaluate the adhesive bond between asphalt binder and aggregate and the cohesive bond between binder molecules. The SFE concept can be applied to select compatible asphalt-aggregate combination(s) based on a satisfactory resistance to moisture damage without the need to performing additional tests (e.g., TSR). It is recommended that highway agencies establish a database of the compatible combinations of the available aggregate and asphalt binders and provide an appropriate strategy to minimize the moisture susceptibility of the asphalt pavement to a satisfactory level by using appropriate adhesion promoters (e.g., hydrated lime or liquid anti-stripping agents) for the incompatible combinations. The existing successful pavements with minimal or no stripping can be used as a reference to establish this database. The minimum cohesive and adhesive bonding between asphalt binder and aggregate can be used to provide a criterion to distinguish between compatible and incompatible combinations based on this concept; hereby, a threshold can be developed based on successfully performing pavements to evaluate different combinations of aggregates and asphalt binders. The importance of this strategy is related to the fact that the practitioners can access the developed database for further implementation and help save cost and time because in this case there is no need to run additional tests to assess the moisture susceptibility of the mixtures for different combinations.

Author Contributions: Aboelkasim Diab wrote the manuscript, while Zhanping You, $\mathrm{Xu}$ Yang, and Mohd Rosli Mohd Hasan took care of the revision.

Conflicts of Interest: The authors declare no conflict of interest.

\section{References}

1. Tarefder, R.A.; Zaman, A. Characterization of Asphalt Materials for Moisture Damage Using Atomic Force Microscopy and Nanoindentation. In Nanotechnology in Civil Infrastructure: A Paradigm Shift; Gopalakrishnan, K., Birgisson, B., Taylor, P., Attoh-Okine, N.O., Eds.; Springer: Berlin/Heidelberg, Germany, 2011; pp. 237-256.

2. Gubler, R.; Partl, M.N.; Canestrari, F.; Grilli, A. Influence of water and temperature on mechanical properties of selected asphalt pavements. Mater. Struct. 2005, 38, 523-532. [CrossRef]

3. Alam, M.M.; Vemuri, N.; Tandon, V.; Nazarian, S.; Picornell, M. A Test Method for Identifying Moisture Susceptible Asphalt Concrete Mixes. Research Project 0-1455: Evaluation of Environmental Conditioning System (ECS) for Predicting Moisture Damage Susceptibility of HMAC; The University of Texas at El Paso: El Paso, TX, USA, 1998. 
4. Cho, D.-W.; Kim, K. The mechanisms of moisture damage in asphalt pavement by applying chemistry aspects. KSCE J. Civ. Eng. 2010, 14, 333-341. [CrossRef]

5. Mohd Hasan, M.R.; You, Z.; Porter, D.; Goh, S.W. Laboratory moisture susceptibility evaluation of WMA under possible field conditions. Constr. Build. Mater. 2015, 101, 57-64. [CrossRef]

6. Kiggundu, B.M.; Roberts, F.L. Stripping in HMA Mixtures: State-of-the-Art and Critical Review of Test Methods; National Center for Asphalt Technology: Auburn, AL, USA, 1988.

7. Hamzah, M.; Hasan, M.; van de Ven, M.; Voskuilen, J. Development of Dynamic Asphalt Stripping Machine for Better Prediction of Moisture Damage on Porous Asphalt in the Field. In 7th RILEM International Conference on Cracking in Pavements; Springer: New York, NY, USA; pp. 71-81. [CrossRef]

8. Solaimanian, M.; Fedor, D.; Bonaquist, R.; Soltani, A.; Tandon, V. Simple performance test for moisture damage prediction in asphalt concrete. Assoc. Asph. Paving Technol. AAPT 2006, 75, 345-380.

9. Diab, A.; Sangiorgi, C.; Enieb, M.; You, Z. A conditioning method to evaluate moisture influence on the durability of asphalt mixture materials. Can. J. Civ. Eng. 2016, 43, 943-948. [CrossRef]

10. Epps, J.; Sebaaly, P.E.; Penaranda, J.; Maher, M.R.; McCann, M.B.; Hand, A.J. Compatibility of a Test for Moisture-Induced Damage with Superpave Volumetric Design; National Academy Press: Washington, DC, USA, 2000.

(C) 2017 by the authors. Licensee MDPI, Basel, Switzerland. This article is an open access article distributed under the terms and conditions of the Creative Commons Attribution (CC BY) license (http://creativecommons.org/licenses/by/4.0/). 